\title{
Metabolic changes induced by theta burst stimulation of the cerebellum in dyskinetic Parkinson's disease patients
}

\author{
Livia Brusa $^{\mathrm{a}}$, Roberto Ceravolo ${ }^{\mathrm{b}}$, Lorenzo Kiferle ${ }^{\mathrm{b}}$, Fabrizia Monteleone ${ }^{\mathrm{c}}$, Cesare Iani ${ }^{\mathrm{a}}$, Orazio Schillaci ${ }^{\mathrm{d}, \mathrm{e}}$, \\ Paolo Stanzione ${ }^{\mathrm{f}, \mathrm{g}}$, Giacomo Koch ${ }^{\mathrm{f}, \mathrm{g}, *}$ \\ ${ }^{a}$ UOC Neurologia, S. Eugenio Hospital, Rome, Italy \\ ${ }^{\mathrm{b}}$ Department of Neuroscience, University of Pisa, Pisa, Italy \\ ${ }^{\mathrm{c}}$ Department of Neuroscience, University of Tor Vergata, Rome, Italy \\ ${ }^{\mathrm{d}}$ Department of Biopathology and Diagnostic Imaging, University of Tor Vergata, Rome, Italy \\ e IRCCS Neuromed, Pozzilli, Italy \\ ${ }^{\mathrm{f}}$ Stroke Unit, Policlinico Tor Vergata, Rome, Italy \\ ${ }^{\mathrm{g}}$ Fondazione Santa Lucia IRCCS, Rome, Italy
}

\section{A R T I C L E I N F O}

\section{Article history:}

Received 4 July 2011

Received in revised form

22 August 2011

Accepted 23 August 2011

\section{Keywords:}

Cerebellum

Parkinson's disease

TMS

Dyskinesias

Levodopa

\begin{abstract}
A B S T R A C T
Background: Cerebellar repetitive transcranial magnetic stimulation may be effective in reducing peakdose levodopa induced dyskinesia in Parkinson's disease patients. It was proposed that the antidyskinetic effect could be due to modulation of cerebello-thalamo-cortical pathways. However the neural basis for these clinical effects have not yet been demonstrated.

Methods: We investigated the effects of repeated sessions of cerebellar continuous theta burst stimulation in Parkinson's disease patients with levodopa induced dyskinesia on brain metabolism by means of positron emission tomography scan with fluorodeoxyglucose ( ${ }^{18} \mathrm{~F}-\mathrm{FDG}$ ) to characterize the specific cerebral network activated by cerebellar stimulation in these patients.

Results: We found that five days of bilateral cerebellar continuous theta burst stimulation (cTBS) were effective in reducing levodopa induced dyskinesia. Clinical changes were paralleled by a reduction of ${ }^{18} \mathrm{~F}-\mathrm{FDG}$ metabolism in the cerebellum as revealed by positron emission tomography imaging. We found a global decrease in the metabolism of the bilateral cerebellar hemispheres, and a significant decrease in ${ }^{18}$ F-FDG uptake in correspondence of bilateral dentate nucleus.

Conclusions: Our study demonstrates the antidyskinetic effect of cerebellar cTBS in Parkinson's disease patients with levodopa induced dyskinesia, is paralleled by modulation of the activity of the pathways connecting the cerebellar cortex with the deep cerebellar nuclei, confirming the hypothesis that the motor cerebellar circuit is involved in the generations of levodopa induced dyskinesia.
\end{abstract}

(c) 2011 Elsevier Ltd. All rights reserved.

\section{Introduction}

Long term therapy with levodopa and dopamine agonists in Parkinson's disease (PD) patients is complicated by the development of fluctuations in motor response, such as levodopa induced dyskinesia (LID) [1,2]. Repetitive Transcranial Magnetic Stimulation (rTMS) has been recently put forward as a possible therapeutic tool able to reduce LID in PD. Trains of $1 \mathrm{~Hz}$ rTMS applied either over the supplementary motor area (SMA) or the primary motor cortex (M1)

\footnotetext{
* Corresponding author. Laboratorio di Neurologia Clinica e Comportamentale, Fondazione Santa Lucia IRCCS, Via Ardeatina, 306, 00179 Roma, Italy. Tel./ fax: +390651501388 .

E-mail addresses: g.koch@hsantalucia.it, giakoch@gmail.com (G. Koch).
}

were able to induce a transient reduction in the severity of LID, suggesting that an over-activity of these areas plays a crucial role in the pathophysiology of LID [3-7]. However, repeated sessions of rTMS were not effective in inducing persistent beneficial clinical effects [4]. Functional or metabolic changes have been reported in the cerebellum in studies in PD patients treated with procedures known to alleviate LID, such as deep brain stimulation (DBS) or pallidotomy of the Globus Pallidus (GPi) [8-10]. Furthermore a recent PET study showed in a sample of PD patients that underwent stereotactic pallidotomy, that the level of binding potential of cerebellar sigma-receptors did not correlate with Hoehn and Yahr ( $\mathrm{H} \& \mathrm{Y}$ ) stages and the Unified Parkinson's Disease Rating Scale (UPDRS), but a strong positive correlation was seen between the binding potential and the preoperative LID severity score, suggesting that cerebellar sigma-receptors may potentially involve the 
genesis of LID in advanced PD [11]. Therefore, the effects of rTMS applied over the lateral cerebellum have been recently tested in patients with LID. A two-week course of bilateral cerebellar continuous theta burst stimulation (cTBS), a novel form of rTMS, induced persistent clinical beneficial effects, reducing peak-dose LID for up to four weeks after the end of the daily stimulation period [12]. Moreover cerebellar cTBS changed the profile of activation of intracortical circuits in the contralateral primary motor cortex (M1), reflecting the long lasting modulation of motor cortical excitability driven by activation of cerebello-thalamo-cortical pathways [13,14]. Thus the effects of cerebellar cTBS were hypothesized to depend on the above described profile of motor cortical excitability, that could represent the cortical reorganization that is associated with a reduction of LID. However, cerebellar cTBS may have expressed its antidyskinetic effect through a direct modulation of the excitability of the cerebellar cortex or through remote changes in other interconnected brain areas. Therefore, in the present study, we aimed to investigate the effects of repeated sessions of cerebellar cTBS in dyskinetic PD on brain metabolism by means of PET scan with FDG at the aim to characterize the specific cerebral network activated by cerebellar stimulation in these patients.

\section{Materials and methods}

\subsection{Cerebellar CTBS}

Eight advanced PD patients suffering from disabling peak-dose dyskinesias following levodopa ingestion were enrolled (see Table 1). Diagnosis of idiopathic PD was made according with Brain Bank Criteria [15]. Anti-parkinsonian medications producing the best control of PD and LID symptoms were fixed for at least one month prior and during the study. Inclusion criteria were: stable medication dose for 4 weeks, and LID $>25 \%$ of waking hours (item 32 of UPDRS $\geq 2$ ) and bothersome (item $33>2$ ). Exclusion criteria were previous PD surgery and contraindications to rTMS. Patients took regular medications during the study except on days of levodopa challenge test. Informed consent and Ethics Board approval were obtained. Patients were in withdrawal of therapy (Core Assessment Program for Surgical Interventional Therapies [CAPSIT]) and had been fasting since the night before. After overnight medication withdrawal, patients received $125 \%$ of their usual morning levodopa equivalent dose as immediate release levodopa/carbidopa. The assessment in each video-recorded session consisted of a complete UPDRS III and CAPSIT dyskinesia scale [16]. LID was assessed individually in the face, neck, trunk, and right and left upper and lower limbs, and was scored as follows: 0 , none; 1 , mild; 2 , moderate; 3 , severe; and 4, extreme ( $0-28)$. After levodopa administration, UPDRS III and CAPSIT were calculated every $15 \mathrm{~min}$ for $1 \mathrm{~h}(\mathrm{t} 0, \mathrm{t} 15, \mathrm{t} 30, \mathrm{t} 45, \mathrm{t} 60)$. Two blinded raters

\section{Table1}

Clinical characteristics of Parkinson's disease patients.

\begin{tabular}{|c|c|c|c|c|c|c|}
\hline $\begin{array}{l}\text { Patient } \\
\text { No. }\end{array}$ & $\begin{array}{l}\text { Age } \\
\text { (years)/ } \\
\text { Sex }\end{array}$ & $\begin{array}{l}\text { Medication } \\
\text { (mg/day) }\end{array}$ & $\begin{array}{l}\text { Duration of } \\
\text { PD (years) }\end{array}$ & $\begin{array}{l}\text { Duration of } \\
\text { dyskinesia } \\
\text { (years) }\end{array}$ & $\begin{array}{l}\text { UPDRS } \\
\text { score } \\
\text { item } 32\end{array}$ & $\begin{array}{l}\text { UPDRS } \\
\text { score } \\
\text { item } 33\end{array}$ \\
\hline 1 & $70 / F$ & $\begin{array}{l}\text { Levodopa (600) } \\
\text { pramipexole (3) }\end{array}$ & 14 & 7 & 3 & 3 \\
\hline 2 & $62 / \mathrm{M}$ & $\begin{array}{l}\text { Levodopa (650) } \\
\text { Pramipexole (4) }\end{array}$ & 10 & 5 & 2 & 2 \\
\hline 3 & $65 / \mathrm{M}$ & $\begin{array}{l}\text { Levodopa (300) } \\
\text { Amantadine (300) } \\
\text { Pramipexole (4) }\end{array}$ & 13 & 6 & 2 & 2 \\
\hline 4 & $61 / F$ & $\begin{array}{l}\text { Levodopa (1000) } \\
\text { Entacapone (600) } \\
\text { Ropinirole (15) }\end{array}$ & 10 & 6 & 2 & 2 \\
\hline 5 & $67 / \mathrm{M}$ & $\begin{array}{l}\text { Levodopa (1000) } \\
\text { Tolcapone } \mathrm{xxx}\end{array}$ & 5 & 2 & 2 & 2 \\
\hline 6 & $75 / \mathrm{M}$ & $\begin{array}{l}\text { Levodopa ( } 800) \\
\text { Ropinirole ( } 24)\end{array}$ & 13 & 7 & 2 & 2 \\
\hline 7 & $63 / F$ & $\begin{array}{l}\text { Levodopa (900) } \\
\text { Entacapone } 600 \\
\text { Pramipexole (3) }\end{array}$ & 11 & 6 & 2 & 3 \\
\hline 8 & $59 / \mathrm{F}$ & $\begin{array}{l}\text { Levodopa (600) } \\
\text { Pramipexole (4) } \\
\text { Amantadine (300) }\end{array}$ & 12 & 8 & 2 & 2 \\
\hline
\end{tabular}

experts in the field of movement disorders rated videotapes independently to provide CAPSIT and UPDRS scores. Both video raters were blinded to rTMS, and the scores were generated after a consensus was reached comparing the individual scores. A MagStim Super Rapid magnetic stimulator (Magstim Company, Whitland, Wales, UK), connected with a figure-of-eight coil with a diameter of $70 \mathrm{~mm}$ was used to deliver cTBS over the scalp sites corresponding to the lateral cerebellum. The magnetic stimulus had a biphasic waveform with a pulse width of about $300 \mu$ s. During the first phase of the stimulus, the current in the center of the coil flowed toward the handle. Three-pulse bursts at $50 \mathrm{~Hz}$ repeated every $200 \mathrm{~ms}$ for $40 \mathrm{~s}$ (equivalent to continuous theta burst stimulation-cTBS [17] were delivered at $80 \%$ of the active motor threshold (AMT) over the lateral cerebellum (600 pulses)). AMT for the first dorsal interosseus (FDI) was tested over the motor cortex of the hemisphere contralateral to each individual patient's side in which dyskinesias were predominant.

cTBS was applied over the lateral cerebellum using the same scalp co-ordinates ( $1 \mathrm{~cm}$ inferior and $3 \mathrm{~cm}$ left/right to the inion) adopted in previous studies, in which MRI reconstruction and neuronavigation systems showed that cerebellar cTBS in this site predominantly target the posterior and superior lobules of the lateral cerebellum $[18,19]$. Although cerebellar stimulation has been originally performed with a double cone coil [20] we used the figure-of-eight coil, since this approach has been adopted in previous investigations in which cerebellar rTMS was shown to be effective in modulating the excitability of the contralateral motor cortex [13]. The coil was positioned tangentially to the scalp, with the handle pointing superiorly. This orientation is able to modulate contralateral M1 excitability [13]. PD patients were submitted to a one week course of bilateral cerebellar cTBS. There were five days of stimulation (five days per week, Monday to Friday). Cerebellar cTBS was applied daily at the same hour in the morning ( 9 a.m.) for each patient. Two trains of cTBS (80\%AMT, 600 pulses, duration $40 \mathrm{~s}$ ) were applied over the left and right lateral cerebellum with a pause of $2 \mathrm{~min}$ between the two trains. The order of stimulation was pseudo-randomized in each subject in every session. The total duration of every daily session was of approximately $4 \mathrm{~min}$. Sham stimulation was delivered through a $70 \mathrm{~mm}$ figure-of-eight focal coil angled at $90^{\circ}$ with only the edge of the coil resting on the scalp. Stimulus intensity, expressed as a percentage of the maximum stimulator output (MSO), was set only at 40\% AMT for the FDI. This stimulation intensity along with the tilted arrangement of the figure-of-eight focal coil, while ineffective in inducing any cortical activation or unpleasant sensations [21-25], ensures an adequate noise and scalp sensation. Evaluation of dyskinesias and UPDRS was performed on day 1, before starting the first session of stimulation (pre-cTBS) and the Monday after the week of stimulation (post-cTBS), in the same days in which PET scanning was performed. cTBS and sham sessions were performed at least three months apart in order to allow for repeated PET scanning. The order of presentation (cTBS or Sham) was counterbalanced across subjects. Therapy was maintained stable during the entire period of the study.

\subsection{PET}

Each patient was placed supine on a bed and an intravenous line was established. Patients were scanned in off dopaminergic medications. Blood glucose levels were controlled in all patients before tracer injection. After $10 \mathrm{~min}{ }^{18} \mathrm{~F}$-FDG (200 MBq) was i.v. administered in a dimly lit room with minimal background noise. Scanning began 40 min after injection. Scans were acquired using a PET/CT scanner Discovery ST (GE Medical Systems, Milwaukee, WI), following a standardized procedure [26] A scout scan projection was used to center brain acquisition. Then a low-dose $(60 \mathrm{~mA}, 120 \mathrm{kV}) \mathrm{CT}$ scan was performed for attenuation correction of PET data. A 3D FDG PET scan was acquired for $10 \mathrm{~min}$. PET data were reconstructed using FBP, a $128 \times 128$ matrix size and a $25 \mathrm{~cm}$ FOV (pixel size $1.95 \times 1.95 \mathrm{~mm}$ and slice thickness $3.27 \mathrm{~mm}$ ). After reconstruction spatial resolution was approximately $6 \mathrm{~mm}$ full-width at half maximum (FWHM) over all planes. Data were then reoriented along the anterior commissure-posterior commissure line, as best inferred by visual inspection approximating the orientation of the SPM PET template, and thus improving the registration accuracy. Reoriented transverse slices were finally exported to a Windows-based personal computer (Microsoft, Redmond, WA, USA) and converted to the Analyze format using the software package MRIcro (http:// www.sph.sc.edu/comd/rorden/mricro.html). PET scans were performed for each subject in the same experimental paradigm under the following conditions: at baseline (pre-cTBS), and after cerebellar cTBS (post-cTBS); for the same subjects such experimental paradigm was then repeated (at most three months apart) in the sham protocol that included a basal scan cTBS (pre-Sham) and another scan performed after sham cerebellar cTBS (post-Sham).

\subsection{Data analysis}

Non-parametric Wilcoxon test were applied on mean CAPSIT dyskinesia scale and UPDRS scores for each session. For statistical analyses, a $p$ value of $<0.05$ was considered to be significant. Mauchley's test examined for sphericity. The Greenhouse-Geisser correction was used for non-spherical data. PET data analysis. Spatial pre-processing and statistical analysis were performed using the SPM2 software (Institute of Neurology, University College of London, London, U.K.) implemented in Matlab 7.3 (The MathWorks, Inc., Natick, MA). For each patient TMS- 
off FDG PET data were spatially realigned to TMS-on data by using a rigid body transformation as well as sham-TMS-off realigned to sham-TMS-On FDG PET data. Each realigned PET volumes were then spatially normalized into the Montreal Neurological Institute (McGill University, Montreal, Quebec, Canada) standard templates by affine transformation (12 parameters for rigid transformations, zooms, and shears) and nonlinear transformations using the relative mean volume as source. Normalized data were smoothed by convolution with an isotropic Gaussian kernel with a 8-mm full-width at half maximum to increase the signal-to-noise ratio. The effects of global metabolism were removed by normalizing the count of each voxel to the total count of the brain using proportional scaling. The following comparisons (post-cTBS versus pre-cTBS; pre-Sham versus. post-Sham images) were performed by using a paired $t$ test based on 2 contrasts (1-1) to detect any regional increase or decrease in metabolism in relation to the two explored different conditions. At a voxel height threshold that had a probability value $p<0.005$ without a correction for multiple comparisons, clusters consisting of a minimum of 100 contiguous voxels were considered significantly different. Results were displayed on the three orthogonal planes of a MRI template.

\section{Results}

The procedure was well tolerated by the PD patients. No adverse effect was reported. The mean patients' AMT taken with the MagStim Super Rapid magnetic stimulator was $43 \pm 2.4 \%$ MSO. We found in this experiment that bilateral cerebellar cTBS was effective in reducing LIDs when applied with repeated sessions during one week. Wilcoxon test revealed that after one week of bilateral cerebellar cTBS global CAPSIT dyskinesia scale scores were decreased in comparison with baseline pre-cTBS evaluation at t15 $(Z=-2.53$; $p=0.011)$, at $\mathrm{t} 30(Z=-2.72 ; p=0.006), \mathrm{t} 45(Z=-2.69 ; p=0.007)$ and at t60 $(Z=-2.53 ; p=0.011)$ (Fig. 1a). No changes were observed in the patients group submitted to sham cTBS (Fig. 1b). Motor abilities scored by the mean UPDRS section III were not modified by any cTBS condition as revealed by Wilcoxon test. SPM analysis of post-cTBS versus pre-cTBS scans disclosed a change in a cluster of cerebellar domains including bilateral cerebellar hemispheres and
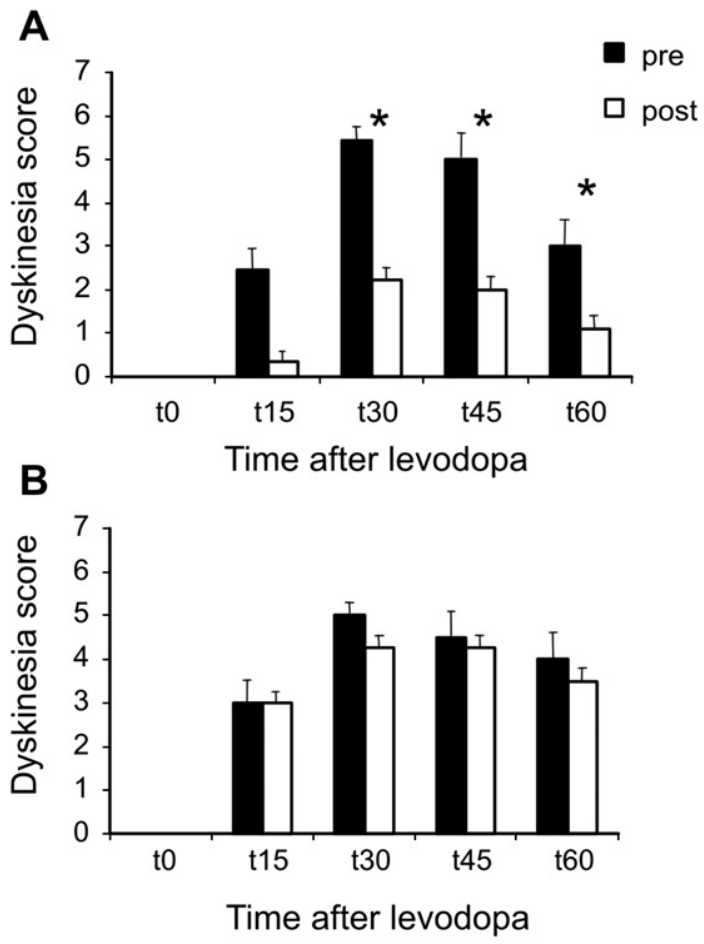

Fig. 1. The graph shows the effect of a one week session of bilateral cerebellar cTBS on LID. Real cTBS (A) but not sham cTBS (B) ameliorates dyskinesia score when tested at different time-points following the administration of $125 \%$ of their usual morning levodopa equivalent dose as immediate release levodopa/carbidopa. Asterisks indicate $p<0.05$. Errors bars indicate S.E.M.

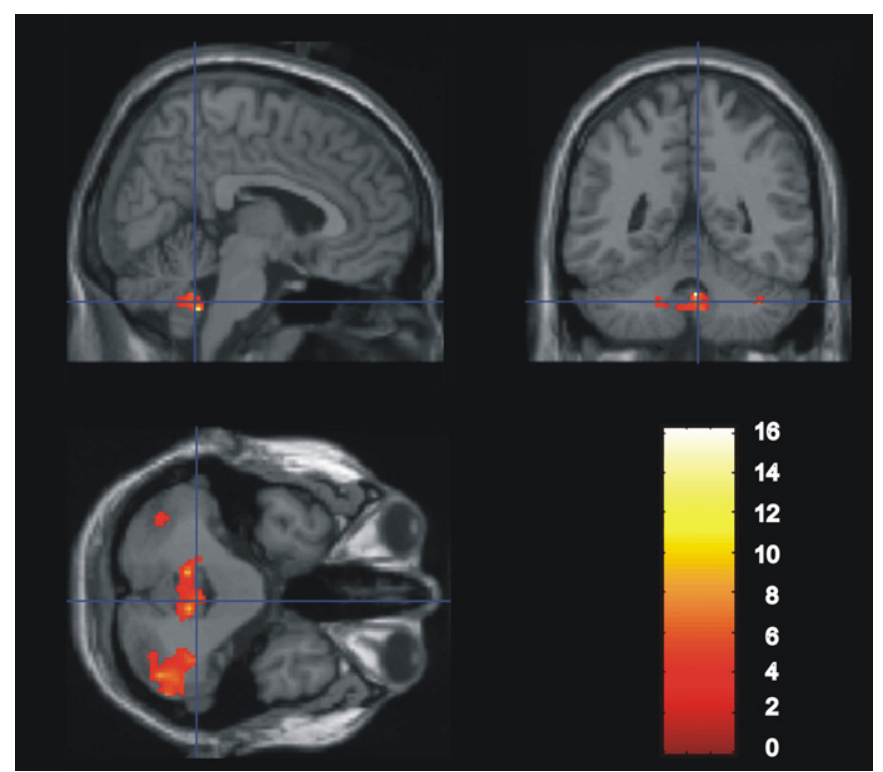

Fig. 2. Regions with significantly decreased cerebral glucose metabolism in the postcTBS versus pre-cTBS scans, measured with SPM2, represented on MRI template including inferior vermis $(46,-68,-34)$ and bilaterally the inferior semilunar lobule $(46,-64,-38$ right; $-51,-65,-38$ left $)$, and dentate nucleus $(10,-54,-30$ right; $-16,-54,-21$ left). $p<0.005$.

the deep cerebellar nuclei. A significant decreased glucose utilization (post-cTBS versus pre-cTBS) was found in a cluster of voxels within inferior vermis $(46,-68,-34)$ and bilaterally in the inferior semilunar lobule $(46,-64,-38$ right; $-51,-65,-38$ left), and dentate nucleus $(10,-54,-30$ right; $-16,-54,-21$ left $)$ (Fig. 2$)$. No changes were detected in other cerebral areas interconnected with the cerebellum. We did not find any significant correlation between the individual changes in glucose utilization in the cerebellar cluster of voxels within the inferior vermis, the inferior semilunar lobules and the dentate nucleus and the decrease of the global CAPSIT dyskinesia scale scores. No significant change (neither increase or decrease) of FDG uptake was observed when post-Sham condition was compared with pre-Sham condition.

\section{Discussion}

The present study confirms and extends our previous results demonstrating the antidyskinetic effect of cerebellar cTBS in PD patients with LID [12]. We found that a week of bilateral cerebellar cTBS was able to induce a reduction of LID; clinical changes were paralleled by a reduction of FDG metabolism in the cerebellum as revealed by PET imaging. Here, we addressed the question of whether cerebellar cTBS may have expressed its antidyskinetic effect through a direct modulation of the cerebellum or through remote changes in other interconnected brain areas. The current findings seem to indicate that cerebellar cTBS exerts its clinical effect through substantial changes in the metabolism of cerebellar areas. In particular we found a global decrease in the metabolism of the bilateral cerebellar hemispheres, with a strong significant decrease in FDG uptake in correspondence of the dentate nuclei. Therefore, it seems likely that cTBS may have modulated the activity of the pathways connecting the cerebellar cortex with the deep cerebellar nuclei. This is consistent with the physiology of the cerebellar-thalamo-cortical pathway activated by magnetic stimulation, that has been recently clarified $[20,27]$. In fact, it has been proposed that cerebellar TMS activates the Purkinje cells of the superior cerebellum; such activation results in an inhibition of the 
dentate nucleus, which is known to exert a background tonic facilitatory drive onto the contralateral motor cortex (M1) through synaptic relay in the ventral lateral thalamus [4]. This in turn leads to an inhibition of the contralateral cerebral cortex, due to a reduction in dentato-thalamo-cortical facilitatory drive [28]. Therefore, our findings seem to confirm the hypothesis that the motor cerebellar circuit is involved in the generations of LIDs [12]. One intriguing possibility is that cerebellar cTBS may have expressed its antidyskinetic effect trough a direct modulation of the excitability of the cerebellar cortex. In analogy with the long term effects of cTBS when applied over the primary motor cortex [17], it is possible that cerebellar cTBS may have induced Long Term Depression (LTD)-like [29] effects in the cerebellar cortex, that could have counteracted an abnormal state of excitability. However, it has to be considered that we were not able to detect changes in other interconnected cerebral areas that were expected to be involved in the LID mechanisms, on the basis of previous imaging and neurophysiological investigations. In fact, LID can be considered the consequence of an abnormal pattern or code of activity that originates and is conveyed from the basal ganglia to the thalamus and the cortical motor areas, leading to overactivation of cortical motor and premotor areas such as the supplementary motor areas, the M1, and the premotor cortex [30-32]. It may well be that these areas indeed might contribute to the onset of LID, but could not be affected by cerebellar stimulation. In alternative, there could be subtle changes in their function that cannot be detected by FDG PET scanning following cerebellar cTBS. For instance there may very well be changes in the motor cortex (or basal ganglia) but changes in cortical inhibition and facilitation may not be detected with FDG PET scan. In addition, it should be noted that there was a slight asymmetry of the metabolic changes induced by cTBS between the cerebellar hemispheres. There were also changes in the vermis, an area of the cerebellum might be more expected to be involved in oculomotor changes rather than those measured in a forelimb muscle. These modifications are not likely to be ascribed to the observed clinical changes. Moreover, it has to be considered that we were not able to demonstrate a direct correlation between the clinical reduction of dyskinesia and the PET metabolic changes that occurred in the cerebellum, given the relatively low number of subjects that took part in the study. Further studies using more specific PET ligands for dopaminergic receptors such as raclopride would allow to deeply investigate remote effects of cerebellar cTBS on the basal ganglia activity [33].

\section{Documentation of author roles}

Research project: LB, GK, PS Conception, OS, PS Organization, FM, OS Execution.

Statistical Analysis: LB, GK, PS Design, LB, GK, RC, LK Execution, PS Review and Critique.

Manuscript Preparation: LB, GK Writing of the first draft, PS, CI, RC, LK Review and Critique.

\section{Disclosure}

The authors have no financial disclosure.

\section{References}

[1] Marsden CD, Parkes JD, Quinn N. Fluctuations of disability in Parkinson's disease-clinical aspects. In: Marsden CD, Fahn S, editors. Movement disorders III. Oxford: Butterworth-Heinemann; 1994. p. 180-99.

[2] Fahn S. The spectrum of levodopa-induced dyskinesias. Ann Neurol 2000;47:2-9.

[3] Koch G, Brusa L, Caltagirone C, Peppe A, Oliveri M, Stanzione P, et al. rTMS of supplementary motor area modulates therapy-induced dyskinesias in Parkinson disease. Neurology 2005;65:623-5.
[4] Brusa L, Versace V, Koch G, Iani C, Stanzione P, Bernardi G, et al. Low frequency rTMS of the SMA transiently ameliorates peak-dose LID in Parkinson's disease. Clin Neurophysiol 2006;117:1917-21.

[5] Wagle-Shukla A, Angel MJ, Zadikoff C, Enjati M, Gunraj C, Lang AE, et al. Lowfrequency repetitive transcranial magnetic stimulation for treatment of levodopa-induced dyskinesias. Neurology 2007;68:704-5.

[6] Filipović SR, Rothwell JC, van de Warrenburg BP, Bhatia K. Slow (1 Hz) repetitive transcranial magnetic stimulation (rTMS) induces a sustained change in cortical excitability in patients with Parkinson's disease. Clin Neurophysiol 2010;121:1129-37.

[7] Rektorova I, Sedlackova S, Telecka S, Hlubocky A, Rektor I. Dorsolateral prefrontal cortex: a possible target for modulating dyskinesias in Parkinson's disease by repetitive transcranial magnetic stimulation. Int J Biomed Imaging 2008;2008:372125.

[8] Starr PA, Vitek JL, Bakay RA. Ablative surgery and deep brain stimulation for Parkinson's disease. Neurosurgery 1998;43:989-1015.

[9] Lang AE. Surgery for levodopa-induced dyskinesias. Ann Neurol 2000; 47(4 Suppl. 1):S193-9. discussion S199-202.

[10] Fukuda M, Mentis MJ, Ma Y, Dhawan V, Antonini A, Lang AE, et al. Networks mediating the clinical effects of pallidal brain stimulation for Parkinson's disease: a PET study of resting-state glucose metabolism. Brain 2001;124: 1601-9.

[11] Nimura T, Ando T, Yamaguchi K, Nakajima T, Shirane R, Itoh M, et al. The role of sigma-receptors in levodopa-induced dyskinesia in patients with advanced Parkinson disease: a positron emission tomography study. J Neurosurg 2004; 100:606-10.

[12] Koch G, Brusa L, Carrillo F, Lo Gerfo E, Torriero S, Oliveri M, et al. Cerebellar magnetic stimulation decreases levodopa-induced dyskinesias in Parkinson disease. Neurology 2009;73:113-9.

[13] Oliveri M, Koch G, Torriero S, Caltagirone C. Increased facilitation of the primary motor cortex following $1 \mathrm{~Hz}$ repetitive transcranial magnetic stimulation of the contralateral cerebellum in normal humans. Neurosci Lett 2005; 376:188-93.

[14] Koch G, Mori F, Marconi B, Codecà C, Pecchioli C, Salerno S, et al. Changes in intracortical circuits of the human motor cortex following theta burst stimulation of the lateral cerebellum. Clin Neurophysiol 2008;119:2559-69.

[15] Hughes AJ, Daniel SE, Kilford L, Lees AJ. Accuracy of clinical diagnosis of idiopathic Parkinson's disease: a clinico-pathological study of 100 cases. J Neurol Neurosurg Psychiatry 1992;55:181-4.

[16] Defer GL, Widner H, Marié RM, Rémy P, Levivier M. Core assessment program for surgical interventional therapies in Parkinson's disease (CAPSIT-PD). Mov Disord 1999;14:572-84.

[17] Huang YZ, Edwards MJ, Rounis E, Bhatia KP, Rothwell JC. Theta burst stimulation of the human motor cortex. Neuron 2005;45:201-6.

[18] Koch G, Oliveri M, Torriero S, Salerno S, Lo Gerfo E, Caltagirone C. Repetitive TMS of cerebellum interferes with millisecond time processing. Exp Brain Res 2007; 179:291-9.

[19] Del Olmo MF, Cheeran B, Koch G, Rothwell JC. Role of the cerebellum in externally paced rhythmic finger movements. J Neurophysiol 2007;98: $145-52$.

[20] Ugawa Y, Uesaka Y, Terao Y, Hanajima R, Kanazawa I. Magnetic stimulation over the cerebellum in humans. Ann Neurol 1995;37:703-13.

[21] Tofts PS. The distribution of induced currents in magnetic stimulation of the nervous system. Phys Med Biol 1990;35(8):1119-28.

[22] Koch G. rTMS effects on levodopa induced dyskinesias in Parkinson's disease patients: searching for effective cortical targets. Restor Neurol Neurosci 2010; 28(4):561-8.

[23] Rothwell JC. Techniques and mechanisms of action of transcranial stimulation of the human motor cortex. J Neurosci Methods 1997;74:113-22.

[24] Wassermann EM, Lisanby SH. Therapeutic application of repetitive transcranial magnetic stimulation: a review. Clin Neurophysiol 2001;112(8): 1367-77.

[25] Strafella A, Ashby P, Lang AE. Pallidotomy increases cortical inhibition in Parkinson's disease. Can J Neurol Sci 1997;24:133-6.

[26] Ceravolo R, Borghetti D, Kiferle L, Tognoni G, Giorgetti A, Neglia D, et al. CSF phosphorylated TAU protein levels correlate with cerebral glucose metabolism assessed with PET in Alzheimer's disease. Brain Res Bull 2008;76:80-4.

[27] Reis J, Swayne OB, Vandermeeren Y, Camus M, Dimyan MA, Harris-Love M, et al. Contribution of transcranial magnetic stimulation to the understanding of cortical mechanisms involved in motor control. J Physiol 2008;586:325-51.

[28] Centonze D, Bernardi G, Koch G. Mechanisms of disease: basic-researchdriven investigations in humans - the case of hyperkinetic disorders. Nat Clin Pract Neurol 2007;3:572-80.

[29] Ito M. Control of mental activities by internal models in the cerebellum. Nat Rev Neurosci 2008;9:304-13.

[30] Brooks DJ, Piccini P, Turjanski N, Samuel M. Neuroimaging of dyskinesia. [Review]. Ann Neurol 2000;47:S154-8.

[31] Rascol O, Sabatini U, Brefel C, Fabre N, Rai S, Senard JM, et al. Cortical motor overactivation in parkinsonian patients with levodopa-induced peak-dose dyskinesia. Brain 1998;121:527-33.

[32] Bezard E, Brotchie JM, Gross CE. Pathophysiology of levodopa-induced dyskinesia: potential for new therapies. Nat Rev Neurosci 2001;2:577-88.

[33] Strafella AP, Paus T, Fraraccio M, Dagher A. Striatal dopamine release induced by repetitive transcranial magnetic stimulation of the human motor cortex. Brain 2003 Dec;126(Pt 12):2609-15. 